\title{
Nurturing Care for Early Childhood Development: Global Perspective and Guidance
}

\author{
Bernadette Daelmans, ${ }^{1}$ Sheila Ashifa Manji, ${ }^{1}$ Neena Raina ${ }^{2}$ \\ From ${ }^{1}$ Child Health and Development Unit; Department of Maternal, Newborn, Child and Adolescent Health and Ageing, World Health \\ Organization, Geneva, Switzerland; ${ }^{2}$ Reproductive, Maternal, Newborn, Child and Adolescent Health and Healthy Ageing, World \\ Health Organization, Regional Office for South-East Asia, New Delhi, India. \\ Correspondence to: Dr Bernadette Daelmans, World Health Organization 20 Avenue Appia, 1211 Geneva 27, Switzerland. \\ daelmansb@who.int
}

To develop to their full potential, all children need to receive nurturing care. This means that, starting in pregnancy, they are raised in a stable environment that is sensitive to their health and nutritional needs, with protection from threats, opportunities for early learning, and interactions that are responsive, emotionally supportive, and developmentally stimulating. Nurturing care ensures the foundations are laid early in life for an individual to survive and thrive. Yet, at least 250 million children younger than 5 years worldwide are at risk of not reaching their developmental potential, having major implications for their health, education, productivity and well-being along the life course. Primary health care services provide a platform for universal support to all families and children, and an entry point for early identification and interventions for families and children with additional needs. Healthcare providers, including pediatricians, are uniquely well placed to watch and learn about the strengths and vulnerabilities of a family and a child, open the dialogue about the child's development, and support caregivers in providing their children nurturing care. Evidence shows that when caregivers are supported to provide all components of nurturing care, starting from pregnancy, children have a better chance to unlock their developmental potential, even when faced with adversities. This paper outlines how the Nurturing Care Framework and its five strategic actions guide multisectoral policies, interventions and services. It articulates the important role the health sector can play in supporting young children's development in the early years.

S urvive, thrive and transform are the objectives of the UN Secretary General's Global Strategy for Women's, Children's and Adolescents' Health (2016-2030) [1]. While substantive progress has been made in tackling under-five mortality, with a reduction in the number of childhood deaths from 12.6 million in 1990 to 5.2 million in 2019 , many children who survive are not able to thrive. Globally, at least 250 million children younger than 5 years are at risk of not reaching their developmental potential, having major implications for their health, education, productivity and well-being along the life course [2]. Poverty, food insecurity, gender inequity, violence, environmental toxins and caregiver's mental health are among the risk factors that affect early childhood development, starting from conception. These risks often coincide at the level of the family and the child, and have cumulative impacts as child-ren grow older [3].

The foundations for an individual's life-long health, cognitive development and social wellbeing are laid down early in life. Within the developing brain, neurons start to form within days of conception and by 4 weeks of gestation up to 250,000 new cells are being formed every minute. In the third trimester of pregnancy, the brain starts to wire itself, making connections between neurons through synapse formation. This wiring is dependent on a supportive environment that provides adequate nutrition, stimulation and protection. If the brain fails to be stimulated appropriately, the formation of neural connections will be impaired, and the brain structure and function will be damaged. Epigenetic changes occur that have negative consequences on a person's abilities and that can be transferred into the next generation.

A solid foundation is laid early in life when children receive nurturing care. This means that they are raised in a stable environment that is sensitive to their health and nutritional needs, with protection from threats, opportunities for early learning, and interactions that are responsive, emotionally supportive, and developmentally stimulating (Fig. 1a) [2,3]. Nurturing care can also restore optimal development when there is adversity. It protects children by lowering their stress levels and encouraging emotional and cognitive coping mechanisms. It is especially important for children with developmental disabilities and is also effective in preventing child maltreatment. Parents and other care-givers are at the center of providing nurturing care for young children. To do so, they need knowledge, time and resources. Supportive communities, services and policies are essential to create the enabling environments that allow caregivers to appropriately care for their young children (Fig. 1b). 

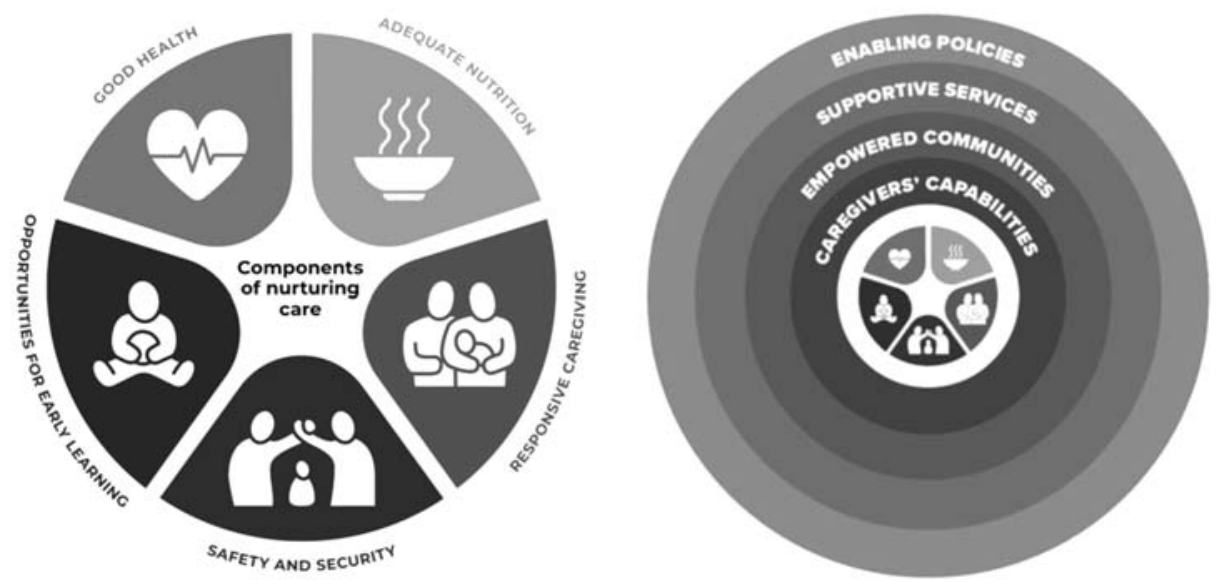

Source: World Health Organization, United Nations Children's Fund, World Bank Group. Nurturing care for early childhood development: a framework for helping children survive and thrive to transform health and human potential. Geneva: World Health Organization; 2018

Fig. 1 ( $a$ ) Components of nurturing care; and $(b)$ Enabling environments for nurturing care [1].

Unfortunately, too few children receive even the most basic interventions to support their optimal development. Globally, the exclusive breastfeeding rate is less than $50 \%$ and almost $22 \%$ of children under five are stunted. In countries with available data, 3 out of 4 children are exposed to violent discipline, and less than half receive responsive care by adults in their home. These risks are distributed across the globe, but they are most prominent in low income countries in South-East Asia and sub-Saharan Africa [4].

\section{THE NURTURING CARE FRAMEWORK}

The Nurturing Care Framework, launched alongside the World Health Assembly in 2018, provides a roadmap for action [5]. It builds on state-of-the art evidence and proposes five strategic actions to facilitate a whole-of-society and whole-of-government approach for every child to receive nurturing care. In so doing, all children will be supported to survive, thrive and reach their full developmental potential. The framework is accompanied by multiple global assets, including illustrative country examples, to help facilitate its operationalization [6].

Strengthening services is one of the five strategic actions of the Nurturing Care Framework and it pertains to systems, workforce and services in health and other sectors. All caregivers need some support for nurturing care, but not all families need the same intensity of support. The framework proposes a three-level approach of universal, targeted, and indicated support (Box I), to meet the needs of all children and their families, with incremental support for those at risk of developmental delays, or with additional needs such as due to developmental disabilities. Identifying the available entry points in contacts that caregivers and young children have with services is a feasible and cost-effective way for getting started to strengthen a three-tiered system of support.

\section{Role of the Health Sector in Supporting Nurturing Care}

From pregnancy to the time children enter formal education, the health sector is uniquely well positioned to support caregivers in providing the five components of nurturing care (Fig. 1). Many interventions that support child survival

Box I Three-tiered System of Support for Early Childhood Development

\section{Universal support}

Antenatal care, childbirth and postnatal care, immunization, and well child and sick child outpatient care are available in all countries and they provide excellent opportunities for supporting nurturing care, including by integrating missing components such responsive caregiving and caregiver support for the general population.

\section{Targeted support}

When families are affected by risks such as poverty, undernutrition, HIV, teenage pregnancy, violence, displacement or humanitarian emergencies, young children are at risk of suboptimal development. They need targeted support that can be provided by home visits, parent groups, and additional facilitybased support. Digital communication can be used to provide caregivers with parenting tips and telephone helplines can assist in solving problems. Targeted support also provides opportunity to link families with other services, like social welfare.

\section{Indicated support}

Globally, an estimated one in six children is living with a developmental disability. Yet, many countries are challenged to provide services, starting with infant and family-centred developmental care in the neonatal period. Investing in workforce capacity and services for indicated support for children with developmental difficulties or disabilities should also be a priority.

Building a three-tiered system: Frontline workers in health facilities and community health workers can be the cornerstone of systems that identify children with developmental disabilities and provide interventions early. 
have a direct impact on early childhood development. Quality antenatal care, essential newborn care, kangaroo mother care for premature and low birth weight infants, exclusive breast-feeding and appropriate complementary feeding, immunization, and timely management of childhood illness are all essential. Pre-conception care enables prospective parents to prepare for future parenthood, by adopting healthier lifestyles, accessing essential interventions such as for anemia, and developing stronger health literacy.

Nonetheless, isolated biomedical interventions alone are not enough. Health and nutrition services need to address all components of nurturing care including interventions for responsive caregiving, early learning activities, safety and security, and supports to caregivers (Box II). WHO and UNICEF developed the Care for Child Development (CCD) package to equip health care pro-viders with the knowledge and the skills to support caregivers in responsive caregiving and early learning activities, through age-specific recommen- dations of communication and play with the child. The Caring for the Caregiver (CFC) package is a complementary set of materials focusing on the social and emotional well-being of caregivers. The interventions underpinning $\mathrm{CCD}$ and $\mathrm{CfC}$ have been integrated into health services in multiple settings and government programs across the world; they have helped primary care workers and pediatricians to strengthen their support for families and

Box II Strengthening Caregiver Capacity to Provide Nurturing Care

\section{Responsive caregiving}

Responsive caregiving is about the caregiver's ability to notice, understand, and respond to their child's signals appropriately. Caregivers who are sensitive have frequent mutually enjoyable interactions with their children, which help young children to understand the world around them and to learn about people, relationships and language.

Opportunities for early learning

As soon as babies are born, they begin to learn through their interactions with other people. Daily routines provide ample opportunity for early learning activities. Smiling and eye contact, talking and singing, modelling and imitation, and simple games like "wave bye-bye" support early learning. Playing with everyday household items like cups and pots helps children learn about objects and their functions. Reading books allows children to learn that objects have names, as well as build curiosity, imagination and knowledge.

Caring for the caregiver

An estimated one in five women experiences signs of perinatal depression globally and many women routinely face the stress of caring for resource-constraint households. By applying techniques for management of relationships, stress, conflict and resources, providers can connect with and support caregivers to address their problems, which in turn helps caregivers connect and support their young children. young children. When implemented with quality, they have shown to improve caregiving practices and learning opportunities in the home, protect children from harsh physical punishment, abuse and neglect, and improve children's developmental outcomes [3].

New data have also illustrated that the intelligence quotient scores of adolescents of a cohort of pre-school children who were living in impoverished communities and were raised in a nurturing home environment, were on average 6 points higher, compared to children who did not benefit from this nurturance [7]. Stunted children whose caregivers received support for responsive caregiving and early learning activities, had an estimated $25 \%$ increase in earnings in adulthood compared to those who did not receive the intervention, putting them at par with nonstunted peers [8].

Pediatricians have a critical role to play. They can provide support for all components of nurturing care in their routine practice and engage in transdisciplinary care for those families and children that need indicated support. Pediatricians can watch and learn about the strengths and vulnerabilities of a family and a child, open the dialogue about the child's development, and support caregivers in providing nurturing care for the child. They can ensure that inpatient neonatal and pediatric care services provide a positive experience for children and their caregivers, and that standards for developmentally supportive care are met. This support is important in their interactions with every family and every child, but especially so for children who are at risk of sub-optimal development or have a developmental disability.

\section{FROM EVIDENCE TO IMPLEMENTATION AT SCALE}

The evidence for nurturing care is compelling but at present, many countries are challenged to implement relevant policies and services at scale. Examples of good practice have mostly emanated from high income countries and they have usually been informed by a strong commitment of the government to reduce inequities. The 'Chile grows with you' program - or Chile Crece Contigo - is often cited as an example of a wholeof-government approach to address young children's development. It started small with strengthening antenatal care and progressively invested in other services along the continuum of care while making available social benefits to vulnerable families. Through a high-level governance mechanism, roles of different sectors were clearly defined, and resources made available to each sector, with accountability for results at every level of the system. India's Integrated Child Development Services (ICDS) program is another exemplar of government commitment to early childhood development. While initial efforts focused on children beyond infancy, its reach and impact are currently 
being amplified by the contribution of the National Health Mission that has the capacity to work with families and young children starting from pregnancy and in the first years of life, through home visits and community services. From these and other examples, lessons have been learnt that can inform the five strategic actions.

\section{Strategic Action 1: Lead and Invest}

To improve children's holistic development, inputs are needed across a range of sectors, through policies, services, infrastructure and information. Examples of policies are a minimum wage, universal health coverage, family-friendly policies in the workplace, and affordable childcare services. It is important for governments to set up functional governance and coordination mechanisms, at national and sub-national levels, to facilitate multi-sectoral collaboration, including public and private sector entities. The development of a national policy that reflects a common vision, goals and targets, and specifies the roles of different sectors and stakeholders, has shown to be helpful to accelerate actions in support of early childhood development. Increases in financing are needed in each sector to add activities to ongoing efforts, and efficiency gains can usually be made through improved collaboration. Health care professionals can make an important contribution to these actions, as champions, experts and practitioners. For example, pediatricians and pediatrics associations can advocate for policy change, participate in national technical advisory groups, and be part of the dialogue regarding multisectoral policies and plans for investing in early childhood development.

\section{Strategic Action 2: Focus on Families and Their Communities}

As nurturing care is so embedded within the lives of each family and child, communities themselves can play a major role in creating enabling environments that benefit both caregivers and young children. Understanding the values and strengths of the community and enabling community participation is key for feasibility, effectiveness and sustaina-bility of strategies that address early childhood development. A strong partnership between community and facility-based services is essential for continuity of care. Healthcare professionals at all levels of the system carry responsibility to make this happen. They can link families to existing services in the communities, help cultivate support networks, and identify and bring visibility to local champions.

\section{Strategic Action 3: Strengthen Services}

Strengthening the capacity of the workforce and building the three levels of support requires substantive efforts. The early integration of nurturing care relevant content in preservice education, complemented by in-service training and continuing education of existing work force, requires the availability of a dedicated group of experts who are well trained themselves. Pediatricians can be at the forefront of capacity building, as trainers, mentors and supervisors. But not only is it important to strengthen skills, attention is also needed for how services are organized in a health facility and community, the triage and management of patient flows, and the time that is available for counselling on nurturing care. Coordination between different disciplines is essential, especially for children with additional needs, and pediatricians can be at the center of organizing transdisciplinary care to ensure all such needs are met.

\section{Strategic Action 4: Monitor Progress}

Improving developmental outcomes is the goal and policymakers will be eager to see results. However, the benefits from investing in nurturing care for early childhood development manifest themselves progressively and it can be dangerous to look for quick wins. To develop strategies and approaches that respond to local context and are effective, scalable and sustainable, it is recommended to start small, monitor and learn, and make adaptations in rapid, iterative cycles. Inputs, outputs and outcomes in all five domains of nurturing care need to be tracked and improvements documented, as they are a precursor to changes in children's developmental status.

The ECD working group of the Countdown to 2030 has brought together country profiles with 42 indicators to support the implementation of the Nurturing Care Framework. Available for 193 countries, profiles include the Early Childhood Development Index 2030, which is a global measure to assess progress on SDG target 4.2, indicator 4.2.1 Proportion of children who are develop-mentally on track in health, learning and psychosocial wellbeing, by sex. Population-based surveys, such as the Multiple Indicator Cluster Survey and the Demographic and Health Survey, are important sources of data that can be used to strengthen programming and sustain advocacy, and health professionals need to consult these.

\section{Strategic Action 5: Use Data and Innovate}

Further research is needed to unpack the evidence behind improving early childhood development but rather than investing in efficacy studies, more efforts are needed to conduct effectiveness and transition-to-scale implementation research. This will help countries to develop appropriate models of care that are feasible for implementation at scale, build capacities for implementation, and obtain context-specific cost data. It will also support identification of innovative solutions to increase access, quality and coverage of services. Point-of-care quality improvement approaches in pediatric health services can make an important contribution in this respect, as exemplified by the 


\section{KEY MESSAGES}

- Early childhood lays the foundation for health, well-being, learning and productivity throughout the life course.

- To optimally grow and develop, children need to receive nurturing care and caregivers need to be supported to provide it, starting from pregnancy.

- National policies, interventions and services are needed to create the enabling environments for parents and other primary caregivers to provide nurturing care.

- The health sector has an important role to play and pediatric services are well placed to facilitate adequate care for all children, and particularly those with developmental disabilities.

- The COVID-19 pandemic has increased the urgency to invest in nurturing care as it has propelled millions of families into poverty and created a global crisis in learning in childhood.

adoption of national operational guidelines for Family Participatory Care in neonatal care units in India; they should be an integral part of service delivery, be well-documented, and results of successes (and failures) shared through professional networks, communities of practices and scientific journals.

These and other challenges have been addressed in the nurturing care handbook, a guide to support the implemen-tation of the NCF in accordance with the five strategic actions [9].

\section{CONCLUSIONS}

A decade is left for countries to achieve the sustainable development goals. The health and well-being of children is clearly spelt out in targets for poverty reduction, food security, health, education, water and sanitation, clean energy, ending of violence and gender equity. Yet, a global crisis has hit the world with the advent of the COVID-19 pandemic, aggravated by a continuation of conflict and displacement of too many people. AUN Secretary General's report states that "children are not the face of COVID-19 pandemic, but they risk being among its biggest victims" [10]. It alerts that 42-66 million children were expected to fall into extreme poverty as a result of the crisis in 2020 alone, adding to the estimated 386 million children already living in extreme poverty in 2019. Virtually every country now faces challenges in making available high quality, equitable and culturally appropriate services that support early childhood development. Giving children what they need to develop is the only way to develop human capital, reduce inequities and realize human rights. Amid the uncertainty and instability affecting today's world, investing in early childhood development lays the foundations for building successful, resilient and peaceful societies. The Nurturing Care Framework pro-vides a roadmap for action. It addresses a wide range of stakeholders and sectors and articulates the important role that the health sector can play in giving young children the best start in life. By deliberately supporting caregivers in providing nurturing care in the earliest years of a child's life, health professionals can make an important contri-bution to 'building back better' and creating a more sustainable world for all.

Note: The authors are staff members of the World Health Organization. The authors alone are responsible for the views expressed in this paper and they do not necessarily represent the decisions, policy or views of the World Health Organization.

Contributors: BD: conceived the manuscript and developed the first draft; BD, SM: revised sections of the manuscript. All authors approved the final version of manuscript.

Funding: None; Competing interests: None stated.

\section{REFERENCES}

1. United Nations. Global Strategy for Women's Children's and Adolescents Health. United Nations; 2016.

2. Richter LM, Daelmans B, Lombardi J, et al. Investing in the foundation of sustainable development: Pathways to scale up for early childhood development. Lancet. 2017;389:103-18.

3. Britto PR, Lye SJ, Proulx K, et al. Nurturing care: Promoting early childhood development. Lancet. 2017;389:91-102.

4. Lu C, Cuartas J, Fink G, et al. Inequalities in early childhood care and development in low/middle-income countries: 2010-2018. BMJ Global Health. 2020;5:e002314.

5. World Health Organization, United Nations Children's Fund, World Bank Group. Nurturing care for early childhood development: A framework for helping children survive and thrive to transform health and human potential. World Health Organization; 2018.

6. Partnership for Maternal, Newborn and Child Health. Nurturing care for early childhood development. Accessed April 01, 2021. Available from: www.nurturing-care.org

7. Trude AC, Richter LM, R Behrman JR, et al. Effects of responsive caregiving and learning opportunities during preschool ages on the association of early adversities and adolescent human capital: An analysis of birth cohorts in two middle-income countries. Lancet Child Adolesc Health. 2021;5:37-46.

8. Gertler P, Heckman J, Pinto R, et al. Labor market returns to an early childhood stimulation intervention in Jamaica. Science. 2014;344: 998-1001.

9. World Health Organization. Nurturing care handbook. Draft for consultation. Accessed April 01, 2021. Available from: https:// nurturing-care.org/handbook/

10. United Nations. Policy Brief: The Impact of Covid-19 on Children. United Nations; 2020. 